\title{
CRANIOTOMIA DESCOMPRESSIVA PARA TRATAMENTO PRECOCE DA HIPERTENSÃO INTRACRANIANA TRAUMÁTICA
}

\author{
Rodrigo Moreira Faleiro', Newton José Godoy Pimenta², Luiz Carlos Mendes Faleiro', \\ Anderson Finotti Cordeiro', Cícero J. Maciel2, Sebastião N.S. Gusmão ${ }^{3}$
}

\begin{abstract}
RESUMO - O papel da craniotomia descompressiva (CD) no tratamento da hipertensão intracraniana (HIC) refratária ainda não está estabelecido na literatura. Atualmente é recomendada como opção, pois há deficiência de trabalhos classe I ou II que suportem seu emprego. Trabalhos recentes têm avaliado a eficácia da CD quando aplicada precocemente no tratamento da HIC pós traumática. No presente trabalho analisamse 21 pacientes nos quais a CD foi realizada precocemente. A maioria dos pacientes apresentava traumatismo cranioencefálico grave (Escala de coma glasgow <9) e tomografia de crânio evidenciando tumefação cerebral (brain swelling) ou hematoma subdural agudo. A principal complicação inerente à técnica foi a hidrocefalia (28,5\%). Boa reabilitação social ocorreu em 11 pacientes $(52,5 \%)$. Parece que a $C D$, quando realizada precocemente, é eficaz no tratamento da HIC refratária, ressaltando-se ainda a necessidade de estudos classe I e II que suportem esta opinião.
\end{abstract}

PALAVRAS-CHAVE: traumatismo cranioencefálico, hipertensão intracraniana, craniotomia descompressiva.

\section{Decompressive craniotomy for the early treatment of traumatic intracranial hypertension}

\begin{abstract}
There is no clear role for decompressive craniotomy (DC) for the intracranial hypertension (ICH) treatment in the literature. Actually, there is a lack of class I or II published data for DC, so it is recomended as a second tier option for the refractory ICH. Recent studies has analized the role of early DC for pos traumatic $\mathrm{ICH}$. The present study analizes 21 patients who has received the early DC for the treatment of traumatic $\mathrm{ICH}$. The majority of the patients had Glasgow Coma Scale $<9$ and harboring a brain swelling or acute subdural hematoma at cranial computadorized tomography. Hydrocephalus was frequent after DC (28.5\%). Good results were obtained in 11 patients (52.5\%). We favour the early application of DC for pos traumatic hypertension.
\end{abstract}

KEY WORDS: head injury, intracranial hypertension, decompressive craniotomy.

A craniotomia descompressiva (CD) é método cirúrgico para redução imediata da pressão intracraniana (PIC). Geralmente é indicada em casos de tumefação cerebral (TCe) e hematoma subdural agudo (HSDA) ou mesmo para lesões não traumáticas'. Consiste em craniotomia unilateral e ampliação do espaço intradural por meio de plástica da dura-máter para se acomodar o cérebro tumefeito. $O$ osso pode ser alojado temporariamente no tecido celular subcutâneo abdominal, congelado (banco de ossos) ou desprezado para posterior cranioplastia com acrílico. Em 1996 foram sistematizadas as condutas em relação ao trauma, classificando-as de acordo com evidências científicas em diretrizes ou opções para o atendimento ao traumatismo crânio-encefálico
$(\mathrm{TCE})^{2}$. Estas diretrizes foram atualizadas em 2000, com a CD sendo sugerida como segunda opção ('se cond tier') para o tratamento da hipertensão intracraniana (HIC), mas sem validação por trabalhos classe I.

A CD foi amplamente utilizada nas décadas de 70 e 80 , mas com resultados desanimadores. Era aplicada apenas quando o tratamento clínico específico de HIC falhava ${ }^{3-6}$. Tentou-se então tratar a HIC por meio de medidas clínicas mais agressivas (coma barbitúrico e hipotermia) que demonstraram eficácia reduzida e complicações clínicas freqüentes ${ }^{5-9}$. Alguns trabalhos recentes avaliaram a eficácia da CD efetuada precocemente nos pacientes vítimas de TCE grave e com HIC. Observaram-se resultados superiores em relação à aplicação tardia e às me-

${ }^{1}$ Neurociru rgão do Hospital Felício Rocho e Hospital Pronto Socorro João XXIII, Belo Horizonte MG, Brasil; ' 2 Residente de neurocirurgia da FHEMIG e Hospital Felício Rocho, Belo Horizonte MG, Brasil; ${ }^{3}$ Neurocirurgião do Hospital das Clínicas da UFMG e Hospital Luxemburgo, Belo Horizonte MG, Brasil.

Recebido 26 Maio 2004, recebido na forma final 16 Novembro 2004. Aceito 13 Janeiro 2005.

Dr. Rodrigo Moreira Faleiro - Rua Caraça 518 / 201 - 30220-260 Belo Horizonte MG - Brasil. E-mail: rfaleiro@brfree.com.br 
didas clínicas de segunda tentativa (coma barbitúrico e hipotermia) $)^{10-14}$.

O objetivo do presente estudo é avaliar a eficácia da CD no tratamento da HIC de origem traumática, quando realizada precocemente.

\section{MÉTODO}

Realizou-se estudo retrospectivo de 21 pacientes vítimas de TCE nos quais a CD foi utilizada precocemente (até 6 horas), durante o período de dois anos (2001-2002), no hospital Pronto Socorro João XXIII. Foram anotados fatores epidemiológicos, Escala de Coma de Glasgow (ECG) inicial e pós-operatória, achados tomográficos e complicações relacionadas à técnica. O seguimento mínimo foi de três meses.

A maioria dos pacientes era do sexo masculino ( $85 \%$ ), com média de idade de 31 anos (3-53 anos). A causa mais comum foi o acidente de trânsito $(52,3 \%)$, seguido por quedas $(23,9 \%)$. A maioria dos pacientes apresentava TCE grave $(E C G<9)(62 \%)$. Segundo achados tomográficos, 95\% dos pacientes apresentavam TCE com desvio de linha média, dos quais $71 \%$ tinham HSDA associado.

Em decorrência do número limitado de leitos no Centro de Tratamento Intensivo (CTI) e de monitores para medida da PIC, na maioria dos casos a indicação para se re alizar a CD baseou-se nos sinais indiretos de HIC vistos à tomografia computadorizada (TC) (desvio da linha média, apagamento dos sulcos e cisternas basais e desaparecimento do terce iroventrículo). Um paciente, no qual estes sinais não eram definitivos, foi admitido no CTI e submetido a um protocolo de tratamento precoce da PIC (Anexo 1).

Os pacientes foram operados sob anestesia geral, em posição supina e com a cabeça virada para o lado oposto à lesão. A incisão foi ampla e em 'ponto de interrogação invertido' (Becker) para expor as regiões frontal, temporal e parietal. Rebateu-se o couro cabeludo, preservandose a gálea subjacente, para posterior duroplastia. Realizou-se hemicraniectomia ampla, com orifícios de trépano e serra de Gigli, expondo-se os lobos frontal, temporal

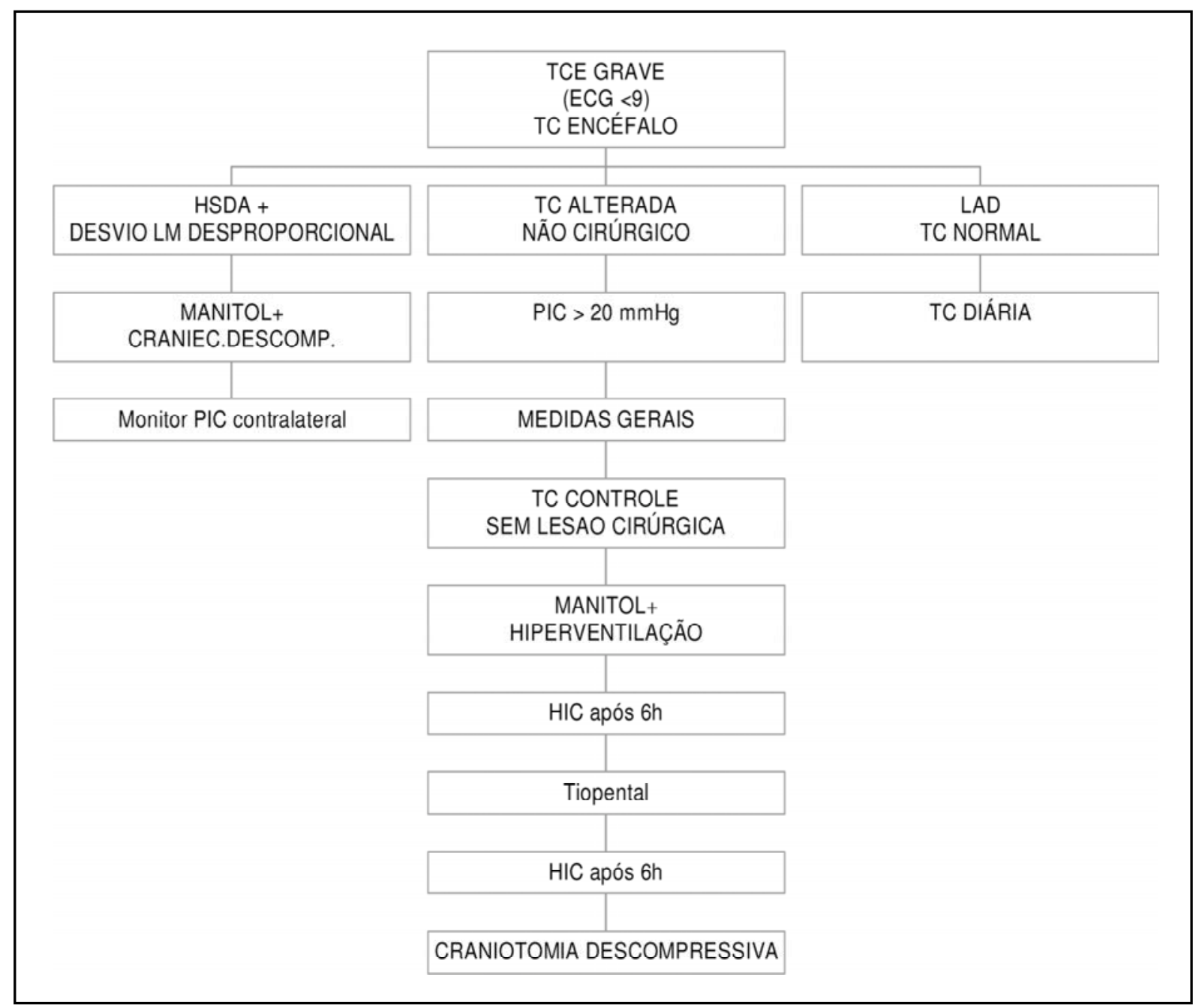

Anexo 1. Protocolo para tratamento precoce da hipertensão intracraniana traumática - HPSJXXIII. LM, linha média; PIC, pressão intracraniana; HIC, hipertensão intracraniana; $L A D$, lesão axonal difusa. 


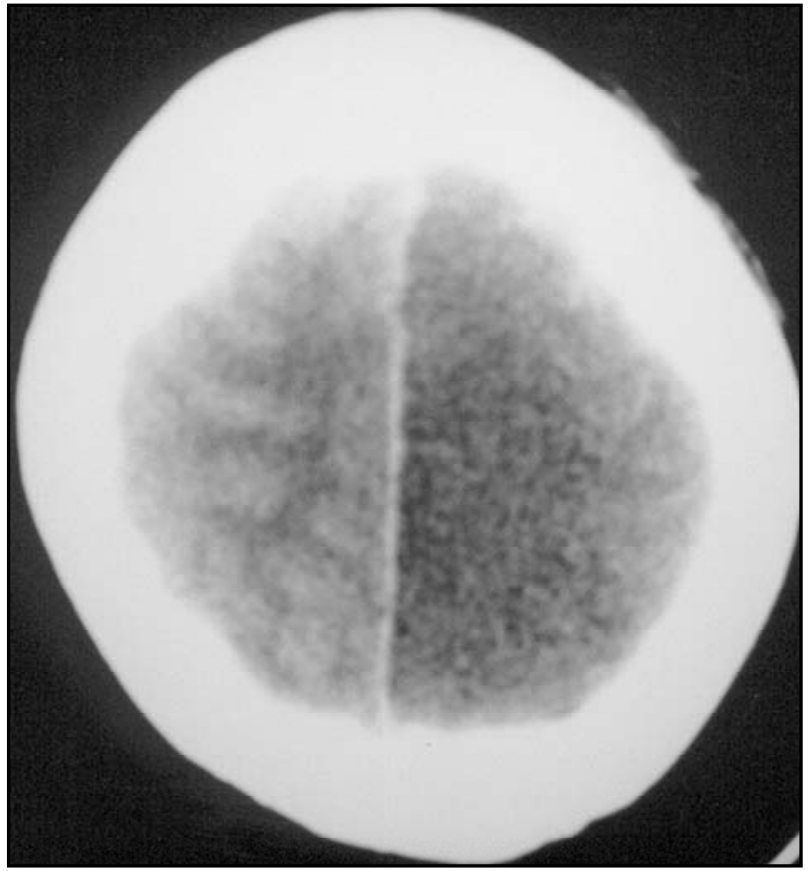

Fig 1. Hipodensidade em hemisfério esquerdo, $2 h$ após trauma cranioencefálico (Tumefação cerebral + isquemia).

e parietal. Ampliou-se a exposição cirúrgica com craniectomia temporal alargada até o assoalho da fossa média e, circunferencialmente, próximo ao seio sagital superior. A seguir realizou-se a abertura da dura-máter. Hematomas extraaxiais e contusões eram drenados e realizouse hemostasia adequada com bipolar e agentes hemostáticos. O procedimento cirúrgico foi completado com a duroplastia e o fechamento por planos (Figs 1, 2, 3).
O retalho ósseo foi armazenado na região do tecido celular subcutâneo abdominal, com anti-sepsia rigorosa e sem tensão na linha de sutura. Recolocava-se precocemente o retalho ósseo. Nos casos em que ocorreu infecção na ferida abdominal, optou-se pela cranioplastia com acrílico, também o mais precoce possível.

\section{RESULTADOS}

Como complicações inerentes à técnica cirúrgica, ocorreu no período de duas a quatro semanas hidrocefalia dependente da válvula em $28,5 \%$ dos casos. O primeiro sinal observado, antes de qualquer alteração neurológica, foi o abaulamento da área de craniectomia. Ocorreram dois casos de infecção de ferida cirúrgica $(9,5 \%)$ e um de meningite $(4,7 \%)$.

A evolução, segundo a Escala de Coma de Glasgow foi: ECG 1 (óbito): 6 casos (28,5\%), ECG 2 e 3 (seqüela moderada/ grave): 4 casos (19\%), ECG 4 (boa reabilitação funcional): 11 casos $(52,5 \%)$.

\section{DISCUSSÃO}

Sabe-se que apenas parte do dano cerebral ocorre no momento do trauma.Vários eventos secundários surgem nas horas e dias seguintes, adicionando efeitos deletérios ao cére bro lesado. Existem vários protocolos que visam evitar ou minimizar estes danos secundários ${ }^{15}$. Tais protocolos enfatizam intubação precoce, transporte rápido e assistido a centro especializado em trauma, reposição volêmica, TC p recoce do encéfalo com imediata evacuação de qualquer massa intracraniana e cuidados intensivos a de-

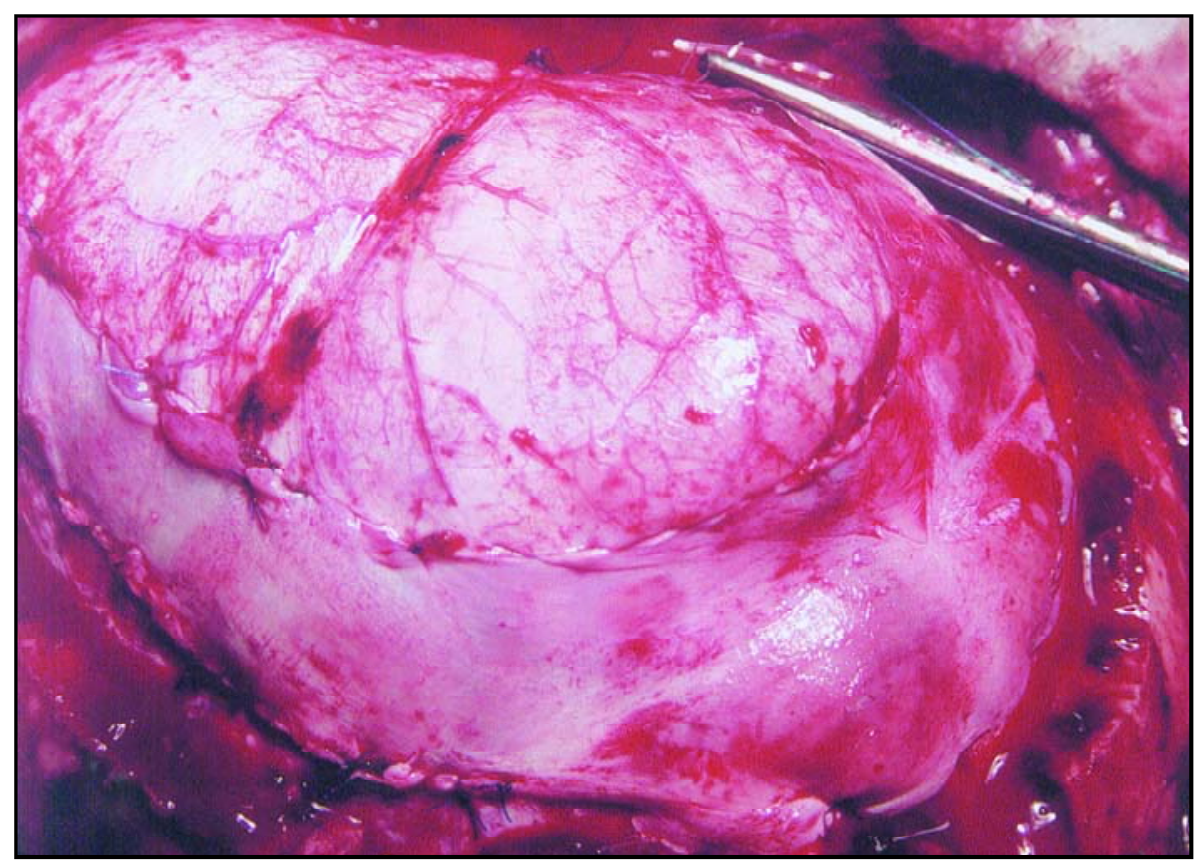

Fig 2. Duroplastia com gálea. 


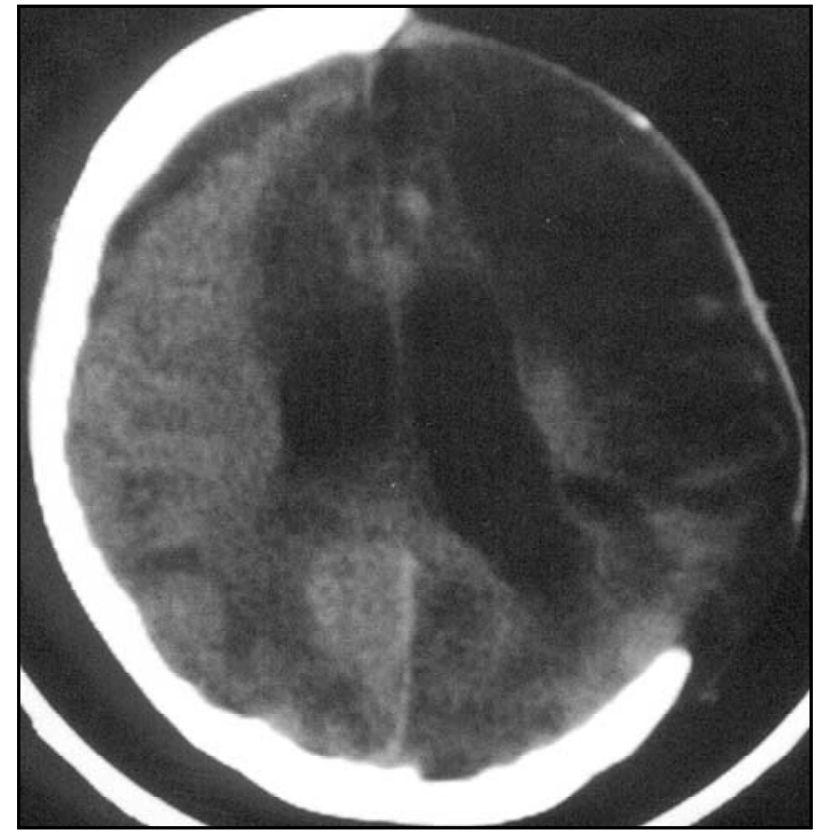

Fig 3. Tomografia de controle, $72 \mathrm{~h}$ após craniotomia descom pressiva.

quados. Tais medidas foram capazes de reduzir a mortalidade no TCE grave de $50 \%$ para $36 \%$, segundo dados do Traumatic Coma Data Bank (TCDB) ${ }^{16}$.

O principal objetivo dos cuidados intensivos no paciente com TCE grave é o de se manter pressão de perfusão cerebral (PPC) adequada enquanto o cérebro se recupera do dano sofrido. Neste sentido, deve-se evitar a hipotensão arterial e a HIC, pois relacionam-se diretamente com a PPC. A PPC é a diferença entre a pressão arterial média (PAM) e a PIC. Em última análise, a HIC vai comprometer a PPC culminando com isquemia e morte neuronal. $A C D$ interfere nesta seqüência de eventos ao permitir a expansão do cérebro. A PPC deve ser mantida acima de $70 \mathrm{~mm} \mathrm{Hg}$ no paciente vítima de TCE grave, o que determina melhor perfusão nas áreas isquêmicas do cérebro lesado e redução da morbidade e mortalidade ${ }^{2}$.

A HIC é por si só um fator de prognóstico negativo. Marmarou et al. ${ }^{16}$ e Stávale ${ }^{17}$ demonstraram que após a idade avançada, alteração do exame motor à admissão e do diâmetro pupilar, a PIC maior que $20 \mathrm{~mm}$ Hg seria o próximo índice de mau pro gnóstico. As medidas para baixar a PIC são classificadas em gerais (manter cabeceira elevada e em posição neutra, evitar vômitos e febre, uso de analgésicos e relaxantes musculares), específicas (sedação intensa, manitol, retirada de líquor e hipenv e ntilação assistida) e de segunda tentativa (coma ba rbitúrico, hipotermia e craniotomia descompressiva).
As medidas gerais são empregadas de rotina, sendo muitas vezes suficientes para a resolução da HIC. Há controvérsias quanto ao tratamento da HIC refratária às medidas gerais e específicas. Sabe-se que $13 \%$ dos pacientes vítimas de TCE grave apresentam HIC refratária ao tratamento convencional (medidas gerais e específicas) ${ }^{5}$, necessitando portanto das medidas de segunda tentativa (hipotermia, coma barbitúrico e $\mathrm{CD}$ ).

Shiozaki ${ }^{7}$ avaliou o resultado da hipotermia moderada em 33 pacientes vítimas de TCE grave, concluindo haver eficácia na redução da PIC e melhora da PPC, com uma maior sobrevida quando comparado ao grupo controle. Posteriormente, outros estudos não evidenciaram melhora estatisticamente significativa no grupo de pacientes com TCE grave submetidos à hipotermia ${ }^{8,9}$. Deve-se monitorizar estes pacientes em uso de hipotermia para se detectar queda no índice cardíaco, trombocitopenia, alteração na função renal e pancreatite ${ }^{18}$.

O coma barbitúrico tem como princípio básico a redução global do metabolismo cerebral, com conseqüente diminuição do compartimento sanguíneo ce rebral e da PIC. É amplamente aplicado na maioria dos centros para tratamento do TCE grave. Relatase que o coma barbitúrico determina hipotensão arterial, com conseqüente comprometimento da PPC, o que por si só é fator de mau prognóstico ${ }^{5,6}$. Schalen et al. ${ }^{6}$ reportaram complicações e efeitos colaterais em 38 pacientes vítimas de TCE grave, medicados com thiopental em altas doses. Encontraram hipotensão arterial em $58 \%$, hipocalemia em $82 \%$, infecções e complicações respiratórias (pneumonia em $53 \%$ ), disfunção hepática em $87 \%$ e disfunção renal em $47 \%$. Relataram mortalidade maior no $g$ rupo que recebeu thiopental associado a dopamina. Esses autores concluíram que outras medidas devem ser empregadas no tratamento da HIC refratária ao tratamento clássico.

Eisenberg et al. ${ }^{5}$, em estudo prospectivo multicêntrico, constataram eficácia do coma barbitúrico no tratamento da HIC. Entretanto, a hipotensão arterial prévia contra-indica a sua aplicação. Relatam ainda a possibilidade de alteração da resposta à $s$ infecções com sepse sem leucocitose ou febre. De acordo com o guidelines for the management of severe head injury, da Associação Americana de Ciru rgiões Neurológicos (AANS) ${ }^{2}$, o coma barbitúrico está contra-indicado como medida profilática de HIC.

Em 1971, Ransohoff et al. ${ }^{3}$, estudaram vítimas de TCE grave e com HSDA. Nos cinco anos anteriores ao referido estudo, a mortalidade dos pacientes com 
HSDA foi $85 \%$. O tratamento realizado era constituído por craniotomia pequena e recolocação do retalho ósseo. Geralmente esses pacientes melhoravam no período de 24 a 72 horas, com piora importante após este período, culminando com alta morbimortalidade. Atribuía-se ao 'edema cerebral' a piora tardia. Na tentativa de melhorar o prognóstico, avaliaram 35 pacientes vítimas de TCE grave e HSDA, nos quais foi realizado, de forma precoce, craniotomia ampla, sem recolocação do retalho ósseo, para se evitar a piora tardia. A mortalidade foi reduzida a $60 \%$, com resultados bons em torno de $28 \%$. Vale ressaltar que todos os pacientes tinham alterações pupilares antes do procedimento cirúrgico, sendo que cinco deles já apresentavam midríase bilateral, o que pode ter alterado negativamente os resultados. Posteriormente, este mesmo grupo publicou resultados sugerindo que a hemicraniectomia descompressiva seria desfavorável, fato co rro borado por Cooper em $1979^{19}$.

Seelig et al. ${ }^{4}$, observaram $57 \%$ de mortalidade geral em 82 pacientes consecutivos tratados de HS DA. Mas quando avaliados aqueles submetidos a cirurgia precoce, antes de 4 horas, a mortalidade foi de $30 \%$. Em $24 \%$ destes pacientes a PIC foi refratária ao tratamento convencional. Metade dos pacientes que faleceram tinha apresentado HIC refratária. Estes pacientes possivelmente seriam beneficiados com o emprego de CD realizada precocemente.

Gower et al. ${ }^{10}$ avaliaram $23 \%$ de pacientes com HIC refratária às medidas convencionais em 115 vítimas de TCE fechado grave (ECG <9). Parte foi submetida a coma barbitúrico, parte a $C D$ subtemporal. O coma barbitúrico foi eficaz na redução da PIC em $30 \%$ dos pacientes, com $82,4 \%$ de mortalidade. A CD controlou a HIC em $70 \%$, com $40 \%$ de mortalidade. É importante notar que quase $30 \%$ do grupo do coma barbitúrico, após falha na redução da PIC foi transferido ao grupo de $C D$, recebendo portanto tratamento 'tardio', fazendo com que tal grupo não fosse homogêneo.

Polin et al. ${ }^{13}$, na tentativa de comparação mais homogênea entre os dois grupos, estudaram retrospectivamente 35 pacientes com TCE refratário ao tratamento convencional e submetidos a CD bifrontal. Utilizaram como grupo controle pacientes tratados por meio de coma barbitúrico extraídos do $\mathrm{TCDB}^{16}$. O grupo cirúrgico teve melhora estatisticamente significativa em relação ao grupo controle submetido a tratamento conservador. A população pediátrica evoluiu melhor e aqueles operados após 48 horas evoluíram desfavoravelmente. Relataram m ortalidade de $23 \%$ e evolução favorável em $37 \%$. Indicam a CD precoce em pacientes com PIC menor que $40 \mathrm{~mm} \mathrm{Hg}$ e com ECG maior que três ${ }^{13}$. Mere ce destaque a ocorrência de dez pacientes $(28,5 \%$ ) que desenvolveram hidrocefalia dependente de derivação ventricular após a $C D$.

Whitfield e Guazzo ${ }^{11}$ descreveram um caso de craniotomia bifrontal realizada precocemente em criança de 13 anos, com bom resultado. Concluíram que a técnica é eficaz no tratamento da HIC refratária às medidas convencionais, sendo indicada em casos de TCE moderado, com piora devida à HIC, desde que não exista lesão primária grave e irreversível.

Guerra et al. ${ }^{12}$ avaliaram 57 pacientes submetidos à CD (26 pacientes com craniectomia bilateral e 31 com craniectomia unilateral). Obtiveram resultados melhores que as séries anteriores, provavelmente pela seleção rigorosa dos candidatos e pela realização precoce da $\mathrm{CD}$. Relataram mortalidade de $19 \%$ com boa reabilitação funcional em $58 \%$ dos casos. A ECG e a PIC no primeiro dia pós-operatório foram os indicadores prognósticos mais importantes. Como complicações tiveram $14 \%$ de hidrocefalia, $26 \%$ de higroma, $7 \%$ de crise convulsiva pósoperatória e $2 \%$ de infecção. Não houve mortalida de diretamente relacionada à técnica. Indicam a $C D$ em pacientes com idade inferior a 50 anos, com sinais de TCE na TC, sem lesão primária irreversível e com HIC refratária ao tratamento convencional ou responsável pela piora neurológica. Munch et al. ${ }^{14}$ avaliaram retrospectivamente 49 pacientes submetidos à $C D$ unilateral, pareando-os com grupo controle de coma barbitúrico provenientes do TCDB. O prognóstico avaliado pela ECG à alta do CTI foi semelhante nos dois grupos, mas com melhora significativa no grupo cirúrgico quando avaliados após seis meses. Neste grupo, os pacientes com idade inferior a 50 anos e operados precocemente evoluíram melhor. $O$ aparecimento da cisterna mesencefálica na TC correlaciona-se melhor com o prognóstico, mesmo quando o desvio da linha média encontrava-se inalterado. Obtiveram $20 \%$ de bons resultados, com 33\% de mortalidade.

Chang et al. ${ }^{20}$ realizam de rotina a CD no TCE grave, apesar da falta de estudos classe I que validem essa conduta. Encontraram vários estudos classe II e III com resultados favoráveis à técnica. Apontam a necessidade de um estudo prospectivo, pareado e multicêntrico que apóie o benefício da CD.

Na presente série os resultados são semelhantes aos comentados anteriormente, que mostram me- 
Ihora progressiva, observada nos casos com resultados favoráveis (ECG 3 e 4). A ECG à admissão foi o principal fator de mau prognóstico, com $50 \%$ de mortalidade nos pacientes com ECG menor que nove.

Me rece discussão a hidrocefalia que ocorre freqüentemente após a $C D$, com relatos de 14 a $28,5 \%$. No presente estudo, foram diagnosticados 6 (28,5\%) pacientes com hidrocéfalo. Em todos os casos, a hid rocefalia ocorreu entre duas a quatro semanas após a cirurgia e foi evidenciada por abaulamento na área da craniectomia. Estamos conduzindo estudo prospectivo para avaliar a etiologia desta hidrocefalia. É aventada como principal hipótese a falta da calota craniana que parece exercer papel importante na absorção liquórica pelas granulações aracnóides (pulsação contínua contra a calota óssea, direcionando-se o fluxo liquórico para a convexidade).

O presente trabalho sugere a eficácia da CD precoce no tratamento da HIC. Entretanto há ainda a necessidade de trabalhos prospectivos, pareados e multicêntricos avaliando-se não só a eficácia, mas também as indicações deste tratamento cirúrgico.

\section{REFERÊNCIAS}

1. Carter BS, Ogilvy CS, Candia GJ, Rosas HD, Buonanno F. One year outcome after decompressive surgery for massive nondominant hemisferic infarction. Neurosurgery 1997;40:1168-1176.

2. Bullock R, Chessnut RM, Clifton G, et al. Guidelines for the management of severe head injury. The Brain Trauma Foundation (New York), The American Association of Neurological Surgeons (Park Ridge, Illinois), and The Joint Section of Neurotrauma and Critical Care, 1995.

3. Ransohoff J, Benjamin MV, Epstein F. Hemicraniectomy for acute subdural hematoma. J Neurosurg 1971;34:70-71.

4. Seelig JM, Becker DP, Miller JD, Greenberg RP, Wa rdJD, Choi SC. Traumatic acute subdural hematoma: major mortality reduction in comatose patients treated within four hours. N Engl J Med 1981;304:1511-1518.
5. Da Silva JA, Da Silva CE, Sousa MB: Decompression craniotomy in acute brain edema: a propos of 30 operated cases. Arq Neuropsiquiatr 1976 34:232-240.

6. Pereira WC, Neves VJ, Rodrigues Y. Bifrontal decompressive craniotomy as the treatment for severe cerebral edema. Arq Neuropsiquiatr 1977 35:99-111.

7. Eisenberg H, Frankowski R, Contant C. Comprehensive central nervous system trauma centers: high-dose barbiturate control of elevated intracranial pressure in patients with severe head injury. J Neurosurg 1988; 69:15-23.

8. Schalen W, Messeter K, Nordstrom CH. Complications and side effects during thiopentone theraphy in patients with severe head injuries. Acta Anaesthesiol Scand 1992;36:369-377.

9. Shiozaki T, Sugimoto H, Taneda M, et al. Effect of mild hypothermia on uncontrollable intracranial hypertension after severe head injury. J Neurosurg 1993;79:363-368.

10. Clifton GL, Miller ER, Choi SC, et al. Lack of effect of induction of hypothermia after acute brain injury. N. Engl J Med 2001;344:556-563.

11. Narayan RK. Hypothermia for traumatic brain injury: a good idea proved ineffective. N Engl J Med 2001;344:602-603.

12. Gower DJ, Lee Stuart, McWhorter JM. Role of subtemporal decompression in severe closed head injury. Neuro su rgery 1988;23:417-422.

13. Whitfield P, Guazzo E. ICP reduction following decompressive craniotomy. Stroke 1995;26:1125-1126.

14. Guerra WKW, Gaab MR, Dietz H, Mueller JU, Piek J, Fritsch MJ. Surgical decompression for traumatic brain sweelling: indications and results. J Neurosurg 1999;90:187-196.

15. Polin RS, Shaffrey ME, Bogaev CA, et al. J Decompressive bifrontal craniectomy in the treatment of severe refractory posttraumatic cerebral edema. Neurosurgery 1997;411:84-94.

16. Munch E, Horn P, Schurer L, Piepgras A, Paul T, Schmiedek P. Management of traumatic brain injury by decompressive craniectomy. Neurosurgery 2000;47:315-322.

17. American College of Surgeons. Advanced trauma life suport (ATLS) 5.Ed. Chicago, 1993.

18. Marmarou A, Anderson JD, Eisenberg HM, et al. The traumatic coma data bank: monitoring of ICP. In Hoff JT, Betz AL (eds). Intracranial pressure VII. Berlin: Springer-Verlag, 1989:549-551.

19. Stávale MA. Bases da terapia intensiva neurológica. São Paulo. Santos Editora, 1996.

20. Greenberg MS. Handbook of neurosurgery, Third Edition. Lakeland: Greenberg Graphics, 1994.

21. Cooper PR, Rovit RL, Ransohoff J. Hemicraniectomy in the treatment of acute subdural hematoma: a re-appraisal. Surgical Neurol 1976;5:25-29.

22. Chang S, Harrington TR, Petersen SR. Craniectomy for traumatic brain injury. Barrow Quarterly, 2003;19:9-17. 